\title{
Asymptotics and stability for global solutions to the Navier-Stokes equations
}

\author{
Isabelle Gallagher Dragoș Iftimie Fabrice Planchon \\ Résumé \\ On étudie des solutions a priori globales des équations de Navier-Stokes \\ incompressibles en trois dimensions d'espace. On montre qu'elles se com- \\ portent en grand temps comme des solutions petites, et en particulier elles \\ décroissent vers zéro quand le temps tend vers l'infini. En utilisant ce résul- \\ tat, on démontre que l'ensemble des données initiales générant des solutions \\ globales est ouvert.
}

\begin{abstract}
We study a priori global strong solutions of the incompressible NavierStokes equations in three space dimensions. We prove that they behave for large times like small solutions, and in particular they decay to zero as time goes to infinity. Using that result, we prove a stability theorem showing that the set of initial data generating global solutions is open.
\end{abstract}

\section{Introduction}

Let us consider the Navier-Stokes equations in $\mathbb{R}^{3}$,

$$
\left\{\begin{array}{c}
\partial_{t} v-\Delta v+v \cdot \nabla v=-\nabla p \\
\nabla \cdot v=0 \\
v_{\mid t=0}=v_{0}
\end{array}\right.
$$

We recall that $(N S)$ describes the movement of an incompressible fluid, whose velocity is $v$ and whose pressure is $p$; note that one can eliminate $\nabla p$ from the equation by projecting it onto the space of divergence free vector fields. Before explaining the aim of our study, let us make some general comments on $(N S)$. Two different kinds of results exist on the Cauchy problem for that system, linked to two important features of $(N S)$ : conserved quantities and scaling. An easy, formal computation shows that the $L^{2}$ norm of the initial data is conserved, due to the following cancellation for any smooth, divergence free vector field $u$ :

$$
\int_{\mathbb{R}^{3}}(u \cdot \nabla) u \cdot u d x=0 .
$$


Using that conservation, J. Leray [11] proved the following result: if the initial data $v_{0}$ is in $L^{2}$, then there is a global solution $v$ to $(N S)$ associated with the data $v_{0}$, satisfying

$$
\forall t \geq 0, \quad\|v(t)\|_{L^{2}}^{2}+2 \int_{0}^{t}\|\nabla v(s)\|_{L^{2}}^{2} d s \leq\left\|v_{0}\right\|_{L^{2}}^{2} .
$$

That solution is not known to be unique, except in two space dimensions. Now let us turn to scaling considerations. The scaling of $(N S)$ is the following: if $v$ solves $(N S)$ with the data $v_{0}$, then $v_{\lambda}(t, x) \stackrel{\text { def }}{=} \lambda v\left(\lambda^{2} t, \lambda x\right)$ solves $(N S)$ with the data $v_{0, \lambda}(x) \stackrel{\text { def }}{=} \lambda v_{0}(\lambda x)$. It is natural to try and solve $(N S)$ in a scale-invariant function space. The typical result in such a space $X$ is that if $v_{0} \in X$ is a divergence free vector field, then one can solve $(N S)$ on a finite time interval, and the solution is unique. The solution is known to be global only for small initial data (in which case the norm of the solution decays to zero as time goes to infinity). Examples of such spaces are

$$
\dot{H}^{1 / 2} \hookrightarrow L^{3} \hookrightarrow \dot{B}_{p, \infty_{\mid p<+\infty}}^{s_{p}} \hookrightarrow B M O^{-1}
$$

where from now on we define $s_{p} \stackrel{\text { def }}{=}-1+3 / p$. We refer to [6], [9], [5], [3], [12] and [10] for precise statements in those function spaces. For global existence to hold, the point in those "strong solution" theorems is that the smallness assumption enables one to get rid of the non linear term, which can be absorbed by the Laplacian. In the Leray theorem, that non linear term is simply made equal to zero, by means of the energy estimate and the cancellation (1.1). Thus the Leray theorem holds specifically for the Navier-Stokes equations, whereas the "strong solution" theorems hold for a larger class of equations, since the special structure of the bilinear term is not used.

As we shall be using them in the following, let us recall the definition of Besov spaces, using Littlewood-Paley theory.

Definition 1 Let $\phi$ be a function in $\mathcal{S}\left(\mathbb{R}^{3}\right)$ such that $\widehat{\phi}=1$ for $|\xi| \leq 1$ and $\widehat{\phi}=0$ for $|\xi|>2$. Define also $\phi_{j}(x)=2^{3 j} \phi\left(2^{j} x\right)$. Then the frequency localization operators are defined by

$$
S_{j}=\phi_{j} * \cdot \quad \Delta_{j}=S_{j+1}-S_{j} .
$$

Let $f$ be in $\mathcal{S}^{\prime}\left(\mathbb{R}^{3}\right)$. Then $f$ is in $\dot{B}_{p, q}^{s}\left(\mathbb{R}^{3}\right)$ if and only if

- The sum $\sum_{-m}^{m} \Delta_{j}(f)$ converges towards $f$ as a tempered distribution if $s<\frac{3}{p}$ and after taking the quotient by polynomials if not.

- The sequence $2^{j s}\left\|\Delta_{j}(f)\right\|_{L^{p}}$ is in $\ell^{q}$.

Our aim in this paper is to consider a priori global strong solutions of the NavierStokes equations, without assuming any sort of smallness on the initial data (such solutions may or may not exist, unless one imposes some additionnal symmetry on the data and the solution). We are interested in the behaviour for large times of such solutions, as well as in their stability. Before stating the result, let us give the following definition; we refer to [4] for the introduction of that type of space in the 
context of the Navier-Stokes equations. The proposition following that definition is classical, we refer for instance to [8] for a proof.

Definition 2 Let $u$ be in $\mathcal{S}^{\prime}$. We will say that $u \in \widetilde{L^{\rho}}\left([a, b], \dot{B}_{p, q}^{s}\right)$ if and only if

$$
2^{j s}\left\|\Delta_{j} u\right\|_{L^{\rho}\left([a, b], L^{p}\right)}=\varepsilon_{j} \in \ell^{q},
$$

and we define

$$
\|u\|_{\widetilde{L^{\rho}}\left([a, b], \dot{B}_{p, q}^{s}\right)} \stackrel{\text { def }}{=}\left\|2^{j s}\right\| \Delta_{j} u\left\|_{L^{\rho}\left((a, b), L^{p}\right)}\right\|_{\ell^{q}} .
$$

Proposition 1 There is a positive real number $\varepsilon_{0}$ such that the following holds. Let $u_{0} \in \dot{B}_{p, q}^{s_{p}}$ with $1 \leq p, q<+\infty$ be a divergence free vector field. There exists a unique solution to $(N S)$ associated with $u_{0}$, satisfying for some time $T>0$

$$
u \in C^{0}\left([0, T], \dot{B}_{p, q}^{s_{p}}\right) \cap \widetilde{L^{r}}\left([0, T], \dot{B}_{p, q}^{s_{p}+\frac{2}{r}}\right) \quad \forall r \in[1,+\infty] .
$$

Moreover if $\left\|u_{0}\right\|_{\dot{B}_{p, q}^{s_{p}}} \leq \varepsilon_{0}$, then one can take $T=+\infty$.

We shall prove the following theorem.

Theorem 1 Let $u_{0} \in \dot{B}_{p, q}^{s_{p}}$, with $1 \leq p, q<+\infty$ be a divergence free vector field, and suppose its associate solution is global, $u \in C^{0}\left(\mathbb{R}^{+}, \dot{B}_{p, q}^{s_{p}}\right)$, and unique (uniqueness is guaranteed for instance as soon as $u \in \widetilde{L^{r}}$ loc $\left(\mathbb{R}^{+}, \dot{B}_{p, q}^{s_{p}+\frac{2}{r}}\right)$, for some $r \in$ $(2,2 /(1-3 / p)))$. Then

$$
\lim _{t \rightarrow \infty}\|u(t)\|_{\dot{B}_{p, q}^{s_{p}}}=0 .
$$

Let us make a few comments on that result. Theorem 1 means that all global solutions of $(N S)$ are in fact small solutions after some time, and satisfy all the properties linked to small data theory; in particular they decay to zero for large times. As we shall see later on, such a result is obvious if the initial data is supposed to be also in the energy space $L^{2}$. In fact the proof of Theorem 1 shows that everything takes place as though there was finite energy, although initially the data is not of finite energy: the first step of the proof, following an idea of C. Calderón [2], consists indeed in bridging the gap between energy and scaling. Theorem 1 enables one to prove the following stability result.

Theorem 2 Under the assumptions of Theorem 1, there is an $\eta_{0}$ (depending on $p, q$ as well as on $\left.\|u\|_{\widetilde{L^{r}}\left(\mathbb{R}^{+}, \dot{B}_{p, q}^{s_{p}+\frac{2}{r}}\right)}\right)$, such that for any divergence free vector field $v_{0} \in \dot{B}_{p, q}^{s_{p}}$ satisfying $\left\|v_{0}-u_{0}\right\|_{\dot{B}_{p, q}^{s_{p}}} \leq \eta_{0}$, its associate solution $v$ satisfies

$$
v \in C^{0}\left(\mathbb{R}^{+}, \dot{B}_{p, q}^{s_{p}}\right) \cap \widetilde{L^{\rho}}\left(\mathbb{R}^{+}, \dot{B}_{p, q}^{s_{p}+\frac{2}{\rho}}\right) \quad \forall \rho \in[1,+\infty],
$$

with

$$
\sup _{t \geq 0}\|v(t)-u(t)\|_{\dot{B}_{p, q}^{s_{p}}}+\|v-u\|_{\widetilde{L^{r}\left(\mathbb{R}^{+}, \dot{B}_{p, q}^{s_{p}+\frac{2}{r}}\right)}} \leq C_{u}\left\|v_{0}-u_{0}\right\|_{\dot{B}_{p, q}^{s_{p}}},
$$

and where $C_{u}$ depends on $p, q$ and $\|u\|_{\widetilde{L}^{r}\left(\mathbb{R}^{+}, \dot{B}_{p, q}^{s_{p}+\frac{2}{r}}\right)}$.

Note that our results are not stated for the space $B M O^{-1}$; however using a similar method of proof, the same result in the $B M O^{-1}$ case was obtained recently by P. Auscher, S. Dubois and P. Tchamitchian [1]. 


\section{Proof of Theorem 1}

\subsection{Large time asymptotics in an easy case}

To give an idea of the proof of Theorem 1, let us start by considering an easy case: suppose that $u_{0}$ is in the inhomogeneous space $H^{1 / 2}$, and that its associate solution is global in time, $u \in C^{0}\left(\mathbb{R}^{+}, \dot{H}^{1 / 2}\right)$. Since the initial data is of finite energy, it is classical that $u$ is in $L^{\infty}\left(\mathbb{R}^{+}, L^{2}\right) \cap L^{2}\left(\mathbb{R}^{+}, \dot{H}^{1}\right)$. Interpolation yields $u \in$ $L^{4}\left(\mathbb{R}^{+}, \dot{H}^{1 / 2}\right)$, which means that for a large enough time, $u$ is sufficiently small in $\dot{H}^{1 / 2}$ for small data theory to apply. In particular $u$ goes to zero in $\dot{H}^{1 / 2}$ as time goes to infinity.

\subsection{Proof of Theorem 1}

To prove Theorem 1, our aim is to try to use the easy remark made in the above Section 2.1. We indeed have two cases when the asymptotics is known: the case of small initial data, and the case of finite energy solutions. The way to link the two is simply to decompose the initial data in the following way, following an idea of $\mathrm{C}$. Calderón [2] (for weak solutions in $L^{p}$ with $2<p<3$; see also [7] where the method was used to prove global existence of infinite energy solutions in two dimensions):

$$
u_{0}=v_{0}+w_{0} \quad \text { with } \quad v_{0} \in L^{2} \quad \text { and } \quad\left\|w_{0}\right\|_{\dot{B}_{p, q}^{s_{p}}} \leq \varepsilon_{0} .
$$

The idea is now to consider the global solution of $(N S)$ associated with $w_{0}$, which satisfies

$$
\begin{gathered}
w \in C^{0}\left(\mathbb{R}^{+}, \dot{B}_{p, q}^{s_{p}}\right) \cap \widetilde{L^{r}}\left(\mathbb{R}^{+}, \dot{B}_{p, q}^{s_{p}+2 / r}\right) \quad \forall r \in[1,+\infty], \quad t^{1 / 2}\|w(t)\|_{L^{\infty}} \in L^{\infty}\left(\mathbb{R}^{+}\right), \\
\text {and } \lim _{t \rightarrow \infty}\|w(t)\|_{\dot{B}_{p, q}^{s_{p}}}=0 .
\end{gathered}
$$

In particular we have

$$
\sup _{t>0} t^{1 / 2}\|w(t)\|_{L^{\infty}} \leq \varepsilon_{0}
$$

Note that in the following, we will note by $\varepsilon_{0}$ any "small" constant, which can change from line to line.

Now we need to study the (perturbated) equation satisfied by $v \stackrel{\text { def }}{=} u-w$ :

$$
\left\{\begin{aligned}
\partial_{t} v-\Delta v+u \cdot \nabla v+v \cdot \nabla w & =-\nabla p \\
\nabla \cdot v & =0 \\
v(x, 0) & =v_{0}(x) .
\end{aligned}\right.
$$

We know that $v \in C^{0}\left(\mathbb{R}^{+}, \dot{B}_{p, q}^{s_{p}}\right) \cap \widetilde{L^{r}}$ loc $\left(\mathbb{R}^{+}, \dot{B}_{p, q}^{s_{p}+\frac{2}{r}}\right)$ since that result holds for both $u$ and $w$. Now let us suppose that we have proved that

$$
v \in L^{\infty}\left(\mathbb{R}^{+}, L^{2}\right) \cap L^{2}\left(\mathbb{R}^{+}, \dot{H}^{1}\right) .
$$

Then as in Section 2.1, $v$ will be small in $\dot{H}^{1 / 2}$ after a long enough time (hence in $\dot{B}_{p, q}^{s_{p}}$ by Sobolev embeddings) and $u=v+w$ will be small enough in $\dot{B}_{p, q}^{s_{p}}$ for small 
data theory to apply. The key point is therefore to prove that $v$ is of finite energy for a large time (we shall not in fact need the global estimate (2.2), a local estimate for a large enough time will do). We shall skip the details here (we refer to [8] for the proof), and simply sketch the steps of the proof.

The first step consists in the proof of the following lemma, which shows that the solution $v$ is of finite energy for a small time.

Lemma 1 There is a time $T^{*}>0$ such that the function $v$ satisfies

$$
v \in C^{0}\left(\left[0, T^{*}\right], L^{2}\right) \cap L^{2}\left(\left[0, T^{*}\right], \dot{H}^{1}\right) .
$$

The proof of Lemma 1 follows from a fixed point procedure, and we shall omit it (see [8]). It is here that there is some work to be done, as the usual fixed point procedure in a scale-invariant space first has to be adapted due to the presence of $u$ and $w$ in the equation, and then the energy norm has to be propagated.

Once that lemma is proved, one is in position to show that the solution $v$ is of finite energy in fact for all times. The method consists in writing an energy estimate, which is possible since the solution $v$ is for a short time in the energy space by Lemma 1. That energy estimate is in fact quite straightforward if one starts the computation at a small time $t_{0}>0$, so let us give the details here. One writes indeed, for $t_{0}<T^{*}$,

$$
\|v(t)\|_{L^{2}}^{2}+2 \int_{t_{0}}^{t}\|\nabla v(s)\|_{L^{2}}^{2} d s=\left\|v\left(t_{0}\right)\right\|_{L^{2}}^{2}-2 \int_{\mathbb{R}^{3}} \int_{t_{0}}^{t}(v \cdot \nabla w) \cdot v d s d x .
$$

But after an integration by parts and Hölder's inequality, we come up with

$$
\left|\int_{\mathbb{R}^{3}} \int_{t_{0}}^{t}(v \cdot \nabla w) \cdot v d s d x\right| \leq \int_{t_{0}}^{t}\|v(s)\|_{L^{2}}\|\nabla v(s)\|_{L^{2}} \sqrt{s}\|w(s)\|_{L^{\infty}} \frac{d s}{\sqrt{s}} .
$$

But (2.1) implies that $\sqrt{s}\|w(s)\|_{L^{\infty}}$ is uniformly bounded by $\varepsilon_{0}$, so we get

$$
\left|\int_{\mathbb{R}^{3}} \int_{t_{0}}^{t}(v \cdot \nabla w) \cdot v d s d x\right| \leq \frac{1}{2} \int_{t_{0}}^{t}\|\nabla v(s)\|_{L^{2}}^{2} d s+\frac{\varepsilon_{0}^{2}}{2} \int_{t_{0}}^{t}\|v(s)\|_{L^{2}}^{2} \frac{d s}{s} .
$$

Plugging that estimate into (2.3) and using Gronwall's lemma, we infer the following estimate:

$$
\|v(t)\|_{L^{2}}^{2}+\int_{t_{0}}^{t}\|\nabla v(s)\|_{L^{2}}^{2} d s \leq\left\|v\left(t_{0}\right)\right\|_{L^{2}}^{2}\left(\frac{t}{t_{0}}\right)^{\varepsilon_{0}^{2}} .
$$

Now by Sobolev embeddings and interpolation we have

$$
\int_{t_{0}}^{t}\|v(s)\|_{\dot{B}_{p, q}^{s_{p}}}^{4} d s \lesssim \int_{t_{0}}^{t}\|v(s)\|_{\dot{H}^{1 / 2}}^{4} d s \leq \int_{t_{0}}^{t}\|v(s)\|_{L^{2}}^{2}\|\nabla v(s)\|_{L^{2}}^{2} d s
$$

which by the above estimate yields

$$
\left(t-t_{0}\right) \inf _{\left[t_{0}, t\right]}\|v(s)\|_{\dot{B}_{p, q}^{s p}}^{4} \lesssim\left\|v\left(t_{0}\right)\right\|_{L^{2}}^{4}\left(\frac{t}{t_{0}}\right)^{\varepsilon_{0}^{2}} .
$$


So we can write, for all $t \geq t_{0}+1$,

$$
\inf _{\left[t_{0}, t\right]}\|v(s)\|_{\dot{B}_{p, q}^{s_{p}}} \leq C\left(t_{0}\right)\left\|v\left(t_{0}\right)\right\|_{L^{2}} \varepsilon^{\varepsilon_{0}^{2}-1 / 4}
$$

which can be made arbitrarily small for $\varepsilon_{0}<\frac{1}{2}$ and $t$ large enough. It follows that one can find a time $\tau_{0}$ such that $\left\|v\left(\tau_{0}\right)\right\|_{\dot{B}_{p, q}^{s_{p}}} \leq \varepsilon_{0}$ and since $\|w\|_{L^{\infty}\left(\mathbb{R}^{+}, \dot{B}_{p, q}^{s p}\right)} \leq \varepsilon_{0}$ we infer that $\left\|u\left(\tau_{0}\right)\right\|_{\dot{B}_{p, q}^{s p}}$ is smaller than $2 \varepsilon_{0}$. We conclude by the small data theory that

$$
\lim _{t \rightarrow \infty}\|u(t)\|_{\dot{B}_{p, q}^{s p}}^{s_{p}}=0
$$

and the theorem is proved.

\section{Proof of Theorem 2}

This section is devoted to the proof of Theorem 2: consider a divergence free vector field $u_{0}$ in $\dot{B}_{p, q}^{\frac{3}{p}-1}$ generating a global solution $u \in C^{0}\left(\mathbb{R}^{+}, \dot{B}_{p, q}^{\frac{3}{p}-1}\right)$. Under the assumptions of the theorem, one can prove (see [8]) that

$$
u \in \widetilde{L^{\rho}}\left(\mathbb{R}^{+}, \dot{B}_{p, q}^{\frac{3}{p}+\frac{2}{\rho}-1}\right)
$$

for all $\rho \in[1,+\infty]$. It is to prove (3.1) that the result of Theorem 1 is used (to transform the local space-time norms into global ones).

Now let $v_{0}$ be another divergence free vector field in $\dot{B}_{p, q}^{\frac{3}{p}-1}$. Its associate solution, which a priori only has a finite life span, is called $v$, and we have $v \in$ $\widetilde{L^{\rho}}\left([0, T], \dot{B}_{p, q}^{\frac{3}{p}+\frac{2}{\rho}-1}\right)$, for all $\rho \in[1,+\infty]$ and for some time $T>0$. We fix for the rest of the proof some $r \in(2,2 /(1-3 / p))$ so that $s \stackrel{\text { def }}{=} s_{p}+\frac{2}{r} \in\left(0, \frac{3}{p}\right)$. If $w$ is defined by $w \stackrel{\text { def }}{=} v-u$, then it is enough to prove that for $\left\|w_{\mid t=0}\right\|_{\dot{B}_{p, q}^{s_{p}}}$ small enough, we have $w \in C^{0}\left(\mathbb{R}^{+}, \dot{B}_{p, q}^{s_{p}}\right) \cap \widetilde{L^{r}}\left(\mathbb{R}^{+}, \dot{B}_{p, q}^{s}\right)$. The function $w$ satisfies the following system:

$$
\left\{\begin{aligned}
\partial_{t} w-\Delta w+w \cdot \nabla w+u \cdot \nabla w+w \cdot \nabla u & =-\nabla p \\
\nabla \cdot w & =0 \\
w_{\mid t=0} & =u_{0}-v_{0} .
\end{aligned}\right.
$$

The following estimate is the key to the proof of the theorem. We shall however not prove it here, as the proof is slightly tedious, but we refer to [8] for details. We have:

$$
\|w\|_{\widetilde{L^{r^{\prime}}\left([\alpha, \beta], \dot{B}_{p, q}^{s^{\prime}}\right)}} \leq K_{2}\left(\|w(\alpha)\|_{\dot{B}_{p, q}^{s p}}+\|w\|_{\widetilde{L^{r}\left([\alpha, \beta], \dot{B}_{p, q}^{s}\right)}}^{2}+\|w\|_{\widetilde{L^{r}\left([\alpha, \beta], \dot{B}_{p, q}^{s}\right)}}\|u\|_{\left.{\widetilde{L^{r}}\left([\alpha, \beta], \dot{B}_{p, q}^{s}\right)}\right)}\right.
$$

for some constant $K_{2}>1$ and all times $\alpha$ and $\beta$. The constant $s^{\prime}$ is arbitrary in $\left[s_{p}, s\right]$ and $r^{\prime}$ is determined by $s^{\prime}=s_{p}+\frac{2}{r^{\prime}}$.

Now we claim that there exist $N$ real numbers $\left(T_{i}\right)_{1 \leq i \leq N}$ such that $T_{1}=0$ and $T_{N}=+\infty$, satisfying

$$
\mathbb{R}_{+}=\bigcup_{i=1}^{N-1}\left[T_{i}, T_{i+1}\right] \quad \text { and } \quad\|u\|_{\widetilde{L}^{r}\left(\left[T_{i}, T_{i+1}\right], \dot{B}_{p, q}^{s}\right)} \leq \frac{1}{4 K_{2}} \quad \forall i \in\{1, \ldots, N-1\}
$$


Indeed since $q<+\infty$, there exists some integer $M$ such that $u^{M} \stackrel{\text { def }}{=} \sum_{|j| \geq M} \Delta_{j} u$ satisfies

$$
\left\|u^{M}\right\|_{\widetilde{L^{r}}\left(\mathbb{R}^{+}, \dot{B}_{p, q}^{s}\right)} \leq \frac{1}{8 K_{2}}
$$

and to obtain the desired time decomposition for $u-u^{M}$, we use the fact that $r<$ $+\infty$ and that one is only summing over a finite number of $j$ 's. The result (3.3) follows.

Now suppose that

$$
\left\|w_{0}\right\|_{\dot{B}_{p, q}^{s_{p}}} \leq \frac{1}{8 K_{2} N\left(2 K_{2}\right)^{N}}
$$

By time continuity we can define a maximal time $T \in \mathbb{R}^{+} \cup\{\infty\}$ such that

$$
\|w\|_{\widetilde{L^{r}}\left([0, T], \dot{B}_{p, q}^{s}\right)} \leq \frac{1}{4 K_{2}} .
$$

If $T=+\infty$ then the theorem is proved. Suppose now that $T<+\infty$. Then we can define an integer $k \in\{1, \ldots, N-1\}$ such that $T_{k} \leq T<T_{k+1}$, and plugging (3.3) and (3.5) into (3.2) with $s^{\prime}=s$ we get for any $i \leq k-1$

$$
\|w\|_{\widetilde{L^{r}\left(\left[T_{i}, T_{i+1}\right], \dot{B}_{p, q}^{s}\right)}} \leq K_{2}\left\|w\left(T_{i}\right)\right\|_{\dot{B}_{p, q}^{s_{p}}}+\frac{1}{4}\|w\|_{\widetilde{L^{r}\left(\left[T_{i}, T_{i+1}\right], \dot{B}_{p, q}^{s}\right)}}+\frac{1}{4}\|w\|_{\widetilde{L}^{r}\left(\left[T_{i}, T_{i+1}\right], \dot{B}_{p, q}^{s}\right)},
$$

so finally

$$
\|w\|_{\widetilde{L^{r}\left(\left[T_{i}, T_{i+1}\right], \dot{B}_{p, q}^{s}\right)}} \leq 2 K_{2}\left\|w\left(T_{i}\right)\right\|_{\dot{B}_{p, q}^{s_{p}}} .
$$

From relation (3.2) with $s^{\prime}=s_{p}$ we also get

$$
\|w\|_{\widetilde{L^{\infty}}\left(\left[T_{i}, T_{i+1}\right], \dot{B}_{p, q}^{s_{p}}\right)} \leq 2 K_{2}\left\|w\left(T_{i}\right)\right\|_{\dot{B}_{p, q}^{s_{p}}}
$$

which implies in particular that

$$
\left\|w\left(T_{i+1}\right)\right\|_{\dot{B}_{p, q}^{s p}} \leq 2 K_{2}\left\|w\left(T_{i}\right)\right\|_{\dot{B}_{p, q}^{s p}} .
$$

A trivial induction now shows that

$$
\left\|w\left(T_{i}\right)\right\|_{\dot{B}_{p, q}^{s_{p}}} \leq\left(2 K_{2}\right)^{i-1}\left\|w_{0}\right\|_{\dot{B}_{p, q}^{s_{p}}} \quad \forall i \in\{1, \ldots, k-1\},
$$

and we conclude from (3.6) and (3.7) that

$$
\|w\|_{\widetilde{L}^{r}\left(\left[T_{i}, T_{i+1}\right], \dot{B}_{p, q}^{s}\right)} \leq\left(2 K_{2}\right)^{i}\left\|w_{0}\right\|_{\dot{B}_{p, q}^{s_{p}}} \text { and } \quad\|w\|_{\widetilde{L^{\infty}\left(\left[T_{i}, T_{i+1}\right], \dot{B}_{p, q}^{s_{p}}\right)}} \leq\left(2 K_{2}\right)^{i}\left\|w_{0}\right\|_{\dot{B}_{p, q}^{s_{p}}}
$$

for all $i \leq k-1$. The same arguments as above also apply on the interval $\left[T_{k}, T\right]$ and yield

and

$$
\|w\|_{\widetilde{L^{r}\left(\left[T_{k}, T\right], \dot{B}_{p, q}^{s}\right)}} \leq\left(2 K_{2}\right)^{k}\left\|w_{0}\right\|_{\dot{B}_{p, q}^{s p}} \leq\left(2 K_{2}\right)^{N}\left\|w_{0}\right\|_{\dot{B}_{p, q}^{s p}}
$$

$$
\|w\|_{\overparen{L^{\infty}}\left(\left[T_{k}, T\right], \dot{B}_{p, q}^{s p}\right)} \leq\left(2 K_{2}\right)^{k}\left\|w_{0}\right\|_{\dot{B}_{p, q}^{s p}} \leq\left(2 K_{2}\right)^{N}\left\|w_{0}\right\|_{\dot{B}_{p, q}^{s_{p}}}
$$

Finally we have

$$
\begin{aligned}
& \|w\|_{\widetilde{L}^{r}\left([0, T], \dot{B}_{p, q}^{s}\right)} \leq\|w\|_{\widetilde{L}^{r}\left(\left[T_{1}, T_{2}\right], \dot{B}_{p, q}^{s}\right)}+\cdots+\|w\|_{\widetilde{L}^{r}\left(\left[T_{k}, T\right], \dot{B}_{p, q}^{s}\right)} \\
& \leq N\left(2 K_{2}\right)^{N}\left\|w_{0}\right\|_{\dot{B}_{p, q}^{s_{p}}}
\end{aligned}
$$

Under assumption (3.4) this contradicts the maximality of $T$ as defined in (3.5). So the theorem is proved. 


\section{References}

[1] P. Auscher, S. Dubois and P. Tchamitchian. Work in progress.

[2] C. Calderón. Existence of weak solutions for the Navier-Stokes equations with initial data in $L^{p}$. Trans. Amer. Math. Soc., 318(1), 179-200, 1990.

[3] M. Cannone. Ondelettes, paraproduits et Navier-Stokes, Diderot éditeur, Arts et Sciences, 1995.

[4] J.-Y. Chemin and N. Lerner. Flot de champs de vecteurs non lipschitziens et équations de Navier-Stokes. J. Differential Equations, 121(2), 314-328, 1995.

[5] G. Furioli, P. Lemarié-Rieusset, and E. Terraneo. Unicité dans $L^{3}\left(\mathbb{R}^{3}\right)$ et d'autres espaces fonctionnels limites pour Navier-Stokes. Rev. Mat. Iberoamericana, 16(3), 605-667, 2000.

[6] H. Fujita and T. Kato. On the Navier-Stokes initial value problem I, Archive for Rational Mechanics and Analysis, 16, 269-315, 1964.

[7] I. Gallagher, D. Iftimie and F. Planchon. Asymptotics and stability for global solutions to the Navier-Stokes equations. Preprint.

[8] I. Gallagher and F. Planchon. On global infinite energy solutions to the NavierStokes equations in two dimensions. Archive for Rational Mechanics and Analysis, 161, 307-337, 2002.

[9] T. Kato. Strong $L^{p}$ solutions of the Navier-Stokes equations in $\mathbb{R}^{m}$ with applications to weak solutions. Math. Zeit. 187, 471-480, 1984.

[10] H. Koch and D. Tataru. Well-posedness for the Navier-Stokes equations. Adv. Math., 157(1), 22-35, 2001.

[11] J. Leray. Sur le mouvement d'un liquide visqueux remplissant l'espace. Acta Mathematica, 63, 193-248, 1934.

[12] F. Planchon. Global strong solutions in Sobolev or Lebesgue spaces to the incompressible Navier-Stokes equations in $\mathbb{R}^{3}$. Annales de l'Institut Henri Poincaré, 13, 319-336, 1996.

Centre de Mathématiques, UMR 7640,

École Polytechnique,

91128 PALAisEAU

Isabelle.Gallagher@math.polytechnique.fr

IRMAR, UMR 6625,

Université de Rennes 1, Campus de Beaulieu, 35042 RENNES

Dragos.Iftimie@math.polytechnique.fr 
Institut GaliLÉE,

Université PARIS 13,

Avenue J.-B. Clément,

93430 Villetaneuse

fab@math . univ-paris13.fr 